\title{
Reduced Decompositions of Matchings
}

\author{
Lun Lv \\ School of Sciences \\ Hebei University of Science and Technology \\ Shijiazhuang 050018, P.R. China \\ klunlv@gmail.com \\ Sabrina X.M. Pang* \\ College of Mathematics and Statistics \\ Hebei University of Economics and Business \\ Shijiazhuang 050061, P.R. China \\ stpangxingmei@heuet.edu.cn
}

Submitted: Dec 28, 2010; Accepted: Apr 30, 2011; Published: May 8, 2011

Mathematics Subject Classifications: 05A05, 05A19

\begin{abstract}
We give a characterization of matchings in terms of the canonical reduced decompositions. As an application, the canonical reduced decompositions of 12312avoiding matchings are obtained. Based on such decompositions, we find a bijection between 12312-avoiding matchings and ternary paths.
\end{abstract}

\section{Introduction}

A matching on a set $[2 n]=\{1,2, \ldots, 2 n\}$ is a graph on $[2 n]$ in which every vertex has degree one. The set of matchings on $[2 n]$ is denoted by $\mathcal{M}_{n}$. Note that $\left|\mathcal{M}_{n}\right|=(2 n-1) ! !=$ $1 \cdot 3 \cdot 5 \cdots(2 n-1)$. The linear representation of a matching is obtained by drawing $2 n$ points in the plane lying on a horizontal line, and connecting them by $n$ arcs such that each arc connects two of the points and lies above the points. Fig. 1 gives the linear representation of the matching $\{(1,3),(2,4),(5,6)\}$.

In this paper, we always use the canonical sequential form [13] of a matching on the set $[2 n]$, which is a permutation of the multiset $\{1,1,2,2, \ldots, n, n\}$ obtained in the following way. Draw the linear representation of the matching, and label the arcs with the numbers

\footnotetext{
${ }^{*}$ Corresponding author
} 


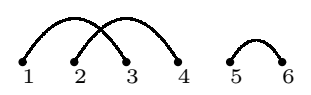

Figure 1: Linear representation.

$1,2, \ldots, n$ ordered by their leftmost endpoints. Then label each endpoint with the label of the adjacent arc, and read the labels of the endpoints from left to right. For example, the matching in Fig. 1 can be also displayed by 121233.

Let $\pi$ and $\tau$ be two sequences. We say $\pi$ avoids $\tau$ or is $\tau$-avoiding, whenever $\pi$ does not contain a subsequence with all of the same pairwise comparisons as $\tau$. For example, the sequence 12342143 is 12123 -avoiding, but not 13132 -avoiding since it has 14143 as a subsequence. In such a context $\tau$ is usually called a pattern. We denote the set of $\tau$-avoiding matchings on $[2 n]$ by $\mathcal{M}_{n}(\tau)$.

The systematic study of pattern avoiding permutations was initiated in 1985 [17]. Starting with the work of Billey, Jockusch and Stanley [3], there has been increasing interest in the connection between reduced decomposition and pattern avoiding permutation (see $[1,2,16,19]$ and references therein). Other results involving pattern avoiding matchings appeared in [5-7, 9-15,20,21].

Recently, by using generating functions, Chen, Mansour and Yan [5] show that the number of 12312-avoiding matchings is given by the 3-Catalan numbers. A combinatorial proof is also given in [5], which is based on a bijection between matchings and oscillating tableaux.

The aim of this paper is to give a new bijective proof for the cardinality of $\mathcal{M}_{n}(12312)$. The idea behind the proof is a new characterization of a matching, which we call the canonical reduced decomposition. In Section 2, we introduce the necessary notations, and describe an algorithm to generate the canonical reduced decomposition of a matching. The canonical reduced decompositions of 12312-avoiding matchings are studied in Section 3. Finally, in Section 4, we apply the canonical reduced decomposition to obtain a bijection between 12312-avoiding matchings and ternary paths. Note that a ternary path is a lattice path in the plane from $(0,0)$ to $(2 n, n)$ with $2 n$ steps $E=(1,0)$ and $n$ steps $N=(0,1)$ and never lying above the line $y=x / 2$.

\section{Canonical reduced decompositions of matchings}

In this section, we characterize matchings in terms of their canonical reduced decompositions. Let $\mathfrak{S}_{n}^{2}$ denote the set of multiset permutations on $\{1,1,2,2, \ldots, n, n\}$. We generalize the notion of reduced decompositions of permutations [19] to multiset permutations.

Definition 2.1. For $1 \leq i \leq 2 n-1$, define a map $s_{i}: \mathfrak{S}_{n}^{2} \rightarrow \mathfrak{S}_{n}^{2}$ such that $s_{i}$ acts on an element $\pi$ in $\mathfrak{S}_{n}^{2}$ by interchanging the integers in positions $i$ and $i+1$. We call $s_{i}$ a simple transposition, and write the action of $s_{i}$ on the right of $\pi$, denoted by $\pi s_{i}$. Therefore, $\pi\left(s_{i} s_{j}\right)=\left(\pi s_{i}\right) s_{j}$. 
For example, $231123 s_{4}=231213$.

Definition 2.2. A reduced decomposition of a multiset permutation $\pi \in \mathfrak{S}_{n}^{2}$ is a sequence of transpositions $s_{i_{0}}, s_{i_{1}}, \ldots, s_{i_{t}}$ such that $\pi=(1122 \cdots n n) s_{i_{0}} s_{i_{1}} \cdots s_{i_{t}}$.

Note that the reduced decomposition of a matching is not unique. For example, $123213=112233 s_{1} s_{2} s_{3} s_{4} s_{3}=112233 s_{2} s_{3} s_{5} s_{4} s_{3}$. To ensure the uniqueness of the decomposition, we give the following definition.

Definition 2.3. A reduced decomposition of a matching $\Lambda$ is canonical if it can be represented by

$$
\Lambda=(1122 \cdots n n) \sigma_{1} \sigma_{2} \cdots \sigma_{k},
$$

where

$$
\begin{aligned}
& \sigma_{i}=s_{h_{i}} s_{h_{i}+1} \cdots s_{t_{i}}, \quad h_{i} \leq t_{i}(1 \leq i \leq k), \\
& h_{i} \in\{2,4, \ldots, 2 n-2\}, \\
& h_{1}>h_{2}>h_{3}>\cdots>h_{k} .
\end{aligned}
$$

In particular, the canonical reduced decomposition of the matching $1122 \cdots n n$ is empty, while the canonical reduced decomposition of the matching $12 \cdots n n \cdots 21$ has the following form

$$
12 \cdots n n \cdots 21=(1122 \cdots n n)\left(s_{2 n-2} s_{2 n-1}\right)\left(s_{2 n-4} s_{2 n-3} s_{2 n-2} s_{2 n-1}\right) \cdots\left(s_{2} s_{3} \cdots s_{2 n-1}\right) .
$$

Theorem 2.4. The canonical reduced decomposition of a matching in $\mathcal{M}_{n}$ is unique.

Proof. We prove the contrapositive: Suppose a matching $\Lambda$ in $\mathcal{M}_{n}$ has two canonical reduced decompositions

$$
\Lambda=(1122 \cdots n n) \sigma_{1} \sigma_{2} \cdots \sigma_{k}=(1122 \cdots n n) \widehat{\sigma}_{1} \widehat{\sigma}_{2} \cdots \widehat{\sigma}_{m},
$$

where $\sigma_{i}=s_{h_{i}} s_{h_{i}+1} \cdots s_{t_{i}}(1 \leq i \leq k)$ and $\widehat{\sigma}_{i}=s_{\widehat{h}_{i}} s_{\widehat{h}_{i}+1} \cdots s_{\widehat{t}_{i}}(1 \leq i \leq m)$.

We shall show $\sigma_{i}=\widehat{\sigma}_{i}$ for any $i$. The first step is to prove $\sigma_{1}=\widehat{\sigma}_{1}$, equivalently, to prove $h_{1}=\widehat{h}_{1}$ and $t_{1}=\widehat{t}_{1}$. We consider the following three cases:

1. $h_{1}>\widehat{h}_{1}$ : The element of $1122 \cdots n n$ in position $h_{1}$ will be transferred to position $t_{1}+1$ by the action of $\sigma_{1}$, that is to say,

$$
(1122 \cdots n n) \sigma_{1}=1122 \cdots\left(\frac{h_{1}}{2}-1\right)\left(\frac{h_{1}}{2}-1\right) \stackrel{\substack{\text { position } \\ \uparrow}}{h_{1}-1} h^{2} \quad\left(\frac{h_{1}}{2}+1\right) \quad \cdots \quad \frac{h_{1}}{2} \quad \cdots .
$$

Since $h_{1}>h_{2}>\cdots>h_{k}$, the action of $\sigma_{2} \cdots \sigma_{k}$ on $(1122 \cdots n n) \sigma_{1}$ preserves the relative order of integers $\frac{h_{1}}{2}, \frac{h_{1}}{2}+1, \ldots, n$. It implies $\Lambda=(1122 \cdots n n) \sigma_{1} \sigma_{2} \cdots \sigma_{k}$ has the subsequence

$$
\frac{h_{1}}{2}\left(\frac{h_{1}}{2}+1\right) \cdots \frac{h_{1}}{2} \cdots .
$$


However, observing that $h_{1}>\widehat{h}_{1}$ and $\widehat{h}_{1}>\widehat{h}_{2}>\cdots>\widehat{h}_{m}$, the matching $\Lambda=$ $(1122 \cdots n n) \widehat{\sigma}_{1} \widehat{\sigma}_{2} \cdots \widehat{\sigma}_{m}$ has the subsequence $\frac{h_{1}}{2} \frac{h_{1}}{2}\left(\frac{h_{1}}{2}+1\right) \cdots$, which gives a contradiction.

2. $h_{1}<\widehat{h}_{1}$ : The proof is similar as Case 1 and we omit it.

3. $h_{1}=\widehat{h}_{1}, t_{1} \neq \widehat{t}_{1}$ : Similar analysis as Case 1 , for $\Lambda=(1122 \cdots n n) \sigma_{1} \sigma_{2} \cdots \sigma_{k}$, the subsequence composed of integers $\frac{h_{1}}{2}, \frac{h_{1}}{2}+1, \ldots, n$ has the form

$$
\frac{h_{1}}{2}\left(\frac{h_{1}}{2}+1\right) \cdots \frac{h_{1}}{2} \cdots,
$$

where there exist $t_{1}-h_{1}+1$ elements between the two appearances of $\frac{h_{1}}{2}$. Meanwhile, for $\Lambda=(1122 \cdots n n) \widehat{\sigma}_{1} \widehat{\sigma}_{2} \cdots \widehat{\sigma}_{m}$, the subsequence composed of integers $\frac{h_{1}}{2}, \frac{h_{1}}{2}+$ $1, \ldots, n$ has the form

$$
\frac{h_{1}}{2}\left(\frac{h_{1}}{2}+1\right) \cdots \frac{h_{1}}{2} \cdots,
$$

and there are $\widehat{t}_{1}-h_{1}+1$ elements between the two appearances of $\frac{h_{1}}{2}$. This contradicts that $t_{1} \neq \widehat{t}_{1}$.

It follows that $\sigma_{1}=\widehat{\sigma}_{1}$. The proof of $\sigma_{i}=\widehat{\sigma}_{i}$ for $i \geq 2$ is analogous.

Note that the product $s_{i} s_{i+1} \cdots s_{j}$ is equivalent to the cyclic permutation on the segment from position $i$ to position $j+1$. For $\Lambda \in \mathcal{M}_{n}$, we describe an algorithm to generate the canonical reduced decomposition of $\Lambda$.

\section{Algorithm:}

1. Let $\Lambda_{1}:=\Lambda$. For $1 \leq i \leq n$, find the position, say $\ell$, of the second appearance of $i$ in $\Lambda_{i}$ :

(1.1) If $\ell=2$, define $\sigma_{n+1-i}$ to be the empty word;

(1.2) If $\ell>2$, define $\sigma_{n+1-i}=s_{2 i} s_{2 i+1} \cdots s_{2 i+\ell-3}$;

(1.3) Generate $\Lambda_{i+1}$ by deleting the two elements $i$ in $\Lambda_{i}$;

2. The canonical reduced decomposition of $\Lambda$ is the product of non-empty words $\sigma_{1}, \sigma_{2}, \ldots, \sigma_{n}$

For example,

$$
\begin{array}{r}
\Lambda_{1}=12331442 \underset{\ell=5}{\stackrel{i=1}{\longrightarrow}} \sigma_{4}=s_{2} s_{3} s_{4}, \Lambda_{2}=233442 \underset{\ell=6}{\stackrel{i=2}{\longrightarrow}} \sigma_{3}=s_{4} s_{5} s_{6} s_{7} \\
\Lambda_{3}=3344 \underset{\ell=2}{\stackrel{i=3}{\longrightarrow}} \sigma_{2} \text { is empty, } \Lambda_{4}=44 \underset{\ell=2}{\stackrel{i=4}{\longrightarrow}} \sigma_{1} \text { is empty. }
\end{array}
$$

Thus, the canonical reduced decomposition of 12331442 is $\left(s_{4} s_{5} s_{6} s_{7}\right)\left(s_{2} s_{3} s_{4}\right)$.

Let $\Lambda^{*}$ be the matching obtained by subtracting 1 from each element of $\Lambda_{2}$. It is constructive to notice the following corollary. 
Corollary 2.5. The canonical reduced decomposition of $\Lambda^{*}$ is the product of non-empty words $\sigma_{1}, \sigma_{2}, \ldots, \sigma_{n-1}$ after subtracting 2 from the index of each simple transposition.

For example, for $\Lambda=12331442$, we have $\Lambda^{*}=122331$ and the canonical reduced decomposition of $\Lambda^{*}$ is $\left(s_{2} s_{3} s_{4} s_{5}\right)$.

Extending the definition of the inversion on permutations [2,4], an inversion of a matching $\pi_{1} \pi_{2} \cdots \pi_{2 n}$ is a pair $\left(\pi_{i}, \pi_{j}\right)$, where $1 \leq i<j \leq 2 n$ and $\pi_{i}>\pi_{j}$.

Corollary 2.6. If $\sigma$ is the canonical reduced decomposition of a matching $\Lambda \in \mathcal{M}_{n}$, then $\Lambda$ has $k$ inversions if and only if $\sigma$ has exactly $k$ simple transpositions.

\section{Canonical reduced decompositions for $\mathcal{M}_{n}(12312)$}

In this section, we restrict the canonical reduced decompositions to 12312-avoiding matchings. We present the following result by inheriting the notations of $\Lambda$ and $\Lambda^{*}$ in the preceding section.

Theorem 3.1. Let $\sigma=\sigma_{1} \sigma_{2} \cdots \sigma_{k}$ be the canonical reduced decomposition of $\Lambda$, where $\sigma_{i}=s_{h_{i}} s_{h_{i}+1} \cdots s_{t_{i}}$ for $1 \leq i \leq k$. Then we have

$$
\Lambda \in \mathcal{M}_{n}(12312) \Leftrightarrow t_{j} \geq t_{i} \text { or } t_{j} \leq h_{i}-2, \quad \text { for } 1 \leq i<j \leq k .
$$

Proof. The cases for $k=0,1$ are trivial. Now we consider $k \geq 2$.

Observe that $\Lambda \in \mathcal{M}_{n}(12312)$ indicates $\Lambda^{*} \in \mathcal{M}_{n-1}(12312)$. We use induction on $n$. Clearly, the statement (3.1) is true for $n=1,2$. By induction hypothesis, we have

$$
\Lambda^{*} \in \mathcal{M}_{n-1}(12312) \Leftrightarrow t_{j}^{*} \geq t_{i}^{*} \text { or } t_{j}^{*} \leq h_{i}^{*}-2, \quad \text { for } 1 \leq i<j \leq m,
$$

where $\sigma_{1}^{*} \sigma_{2}^{*} \cdots \sigma_{m}^{*}$ is the canonical reduced decomposition of $\Lambda^{*}$ and $\sigma_{i}^{*}=s_{h_{i}^{*}} s_{h_{i}^{*}+1} \cdots s_{t_{i}^{*}}$.

For $\Lambda$, let $\ell$ denote the position of the second appearance of 1 . Here are two cases:

1. If $\ell=2$, then $m=k, h_{i}=h_{i}^{*}+2$, and $t_{i}=t_{i}^{*}+2$ for $1 \leq i \leq k$. Moreover, in this case, $\Lambda \in \mathcal{M}_{n}(12312)$ if and only if $\Lambda^{*} \in \mathcal{M}_{n-1}(12312)$. By (3.2), we have

$$
\Lambda \in \mathcal{M}_{n}(12312) \Leftrightarrow t_{j}^{*} \geq t_{i}^{*} \text { or } t_{j}^{*} \leq h_{i}^{*}-2 \Leftrightarrow t_{j} \geq t_{i} \text { or } t_{j} \leq h_{i}-2,
$$

for $1 \leq i<j \leq k$.

2. If $\ell>2$, then $m=k-1, h_{i}=h_{i}^{*}+2, t_{i}=t_{i}^{*}+2$, for $1 \leq i \leq k-1$, and

$$
\sigma_{k}=s_{2} s_{3} \cdots s_{\ell-1}
$$

which gives $h_{k}=2$ and $t_{k}=\ell-1$. In this case, we prove (3.1) in two steps:

Step 1. $(\Leftarrow)$ By $(3.2)$, we get $\Lambda^{*} \in \mathcal{M}_{n-1}(12312)$. So it is sufficient to show that $\Lambda$ does not contain a subsequence

$$
1, \ldots, i_{1}, \ldots, i_{2}, \ldots, 1, \ldots, i_{1}
$$


where $i_{2}>i_{1}>1$. Furthermore, we need only show that $\Lambda$ does not have a subsequence

$$
1, \ldots, \frac{h_{i_{0}}}{2}, \ldots, \frac{h_{i_{0}}}{2}+1, \ldots, 1, \ldots, \frac{h_{i_{0}}}{2}
$$

where $\frac{h_{i_{0}}}{2}+1$ is the first appearance in $\Lambda$. By contradiction, choose a subsequence of the form (3.3) such that $h_{i_{0}}$ is minimal. This implies that the element $\frac{h_{i_{0}}}{2}+1$ in (3.3) is in position $h_{i_{0}}-1$ of $\Lambda$. Notice that the second appearance of 1 in (3.3) is in position $t_{k}+1$ of $\Lambda$, and the position of the second appearance of $\frac{h_{i_{0}}}{2}$ in (3.3) is not after the position $t_{i_{0}}+1$ in $\Lambda$. It follows that $t_{k}+1>h_{i_{0}}-1$ and $t_{k}+1<t_{i_{0}}+1$. Thus, we deduce that $t_{k}>h_{i_{0}}-2$ and $t_{k}<t_{i_{0}}$, which is a contradiction to the right hand side of (3.1).

Step 2. $(\Rightarrow)$ By $(3.2)$, we have $t_{j}^{*} \geq t_{i}^{*}$ or $t_{j}^{*} \leq h_{i}^{*}-2$ for $1 \leq i<j \leq k-1$. This gives $t_{j} \geq t_{i}$ or $t_{j} \leq h_{i}-2$ for $1 \leq i<j \leq k-1$. Then it suffices to prove that $t_{k} \geq t_{i}$ or $t_{k} \leq h_{i}-2$ for $1 \leq i \leq k-1$. Otherwise, choose $i_{0}$ to be the maximal index such that $t_{k}<t_{i_{0}}$ and $t_{k}>h_{i_{0}}-2$. This implies that the second appearance of $\frac{h_{i_{0}}}{2}$ in $\Lambda$ is in position $t_{i_{0}}+1$. Notice that the second appearance of 1 in $\Lambda$ is in position $t_{k}+1$, and the position of the first appearance of $\frac{h_{i_{0}}}{2}+1$ is not after the position $h_{i_{0}}+1$ in $\Lambda$. Therefore, there exists a subsequence of $\Lambda$ with the following form

$$
1, \ldots, \frac{h_{i_{0}}}{2}, \ldots, \frac{h_{i_{0}}}{2}+1, \ldots, 1, \ldots, \frac{h_{i_{0}}}{2}, \ldots
$$

which contradicts that $\Lambda$ is 12312 -avoiding.

\section{Bijection between ternary paths and $\mathcal{M}_{n}(12312)$}

Chen, Mansour and Yan [5] show that the number of 12312-avoiding matchings on [2n] equals the 3-Catalan numbers [18, Sequence A001764], namely,

$$
\left|\mathcal{M}_{n}(12312)\right|=\frac{1}{2 n+1}\left(\begin{array}{c}
3 n \\
n
\end{array}\right) \text {. }
$$

Note that the 3-Catalan numbers also count ternary paths of length $3 n$. A ternary path of length $3 n$ is a lattice path in the plane from $(0,0)$ to $(2 n, n)$ with $2 n$ steps $E=(1,0)$ and $n$ steps $N=(0,1)$ and never lying above the line $y=x / 2$. For example, a ternary path $P=E E E E E N E E E N E E N E N E N N$ is shown in Fig. 2.

The purpose of this section is to establish a bijection between $\mathcal{M}_{n}(12312)$ and ternary paths of length $3 n$. We follow the approach of some known results $[2,8]$ to pattern avoiding permutations. Moreover, our bijection will rely on the canonical reduced decompositions of 12312-avoiding matchings.

By Definition 2.3 and Theorem 3.1, $\sigma_{1} \sigma_{2} \cdots \sigma_{k}$ is the canonical reduced decomposition of $\Lambda \in \mathcal{M}_{n}(12312)$, where $\sigma_{i}=s_{h_{i}} s_{h_{i}+1} \cdots s_{t_{i}}$ for $1 \leq i \leq k$, if and only if the set of 


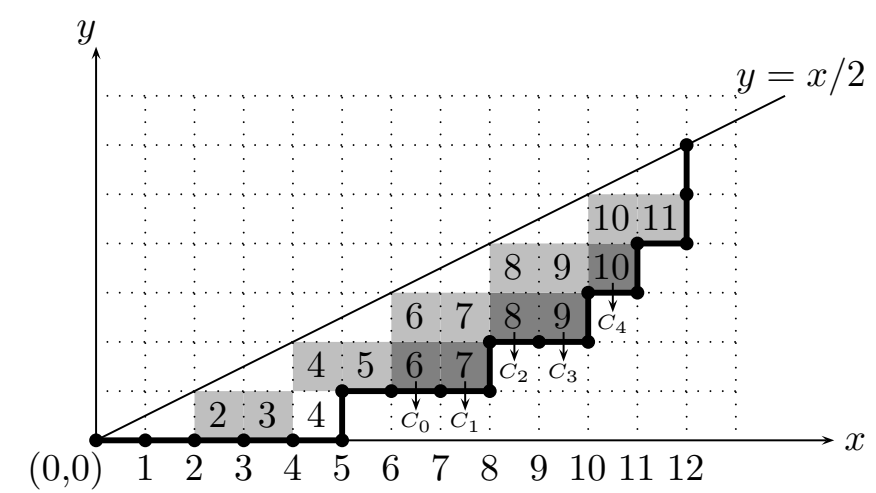

Figure 2: The strip decomposition.

parameters $\left\{\left(h_{i}, t_{i}\right) \mid 1 \leq i \leq k\right\}$ satisfies

$$
\begin{aligned}
& h_{1}>h_{2}>\cdots>h_{k}, \\
& t_{i} \geq h_{i} \in\{2,4, \ldots, 2 n-2\}, \quad(1 \leq i \leq k), \\
& t_{j} \geq t_{i} \text { or } t_{j} \leq h_{i}-2, \quad(1 \leq i<j \leq k) .
\end{aligned}
$$

For a ternary path $P$, our bijection involves all the unit cells enclosed by $P$. Explicitly, a cell enclosed by $P$ means that the cell is totally in the region surrounded by $P$ and $y=x / 2$. We give an $x$-labeling of these cells: Each cell with corner points $(i, j),(i+1, j)$, $(i+1, j+1)$ and $(i, j+1)$, receives a label $i$. We call a cell with an even (resp. odd) label an even cell (resp. odd cell) for short. A cell enclosed by $P$ is self-dependent if the cell immediately to its South-West is not enclosed by $P$. We define the ladder strip of $P$ as follows:

1. If $P=(E E N)^{n}$, that is, $P$ is composed of $n$ consecutive segments $E E N$, then $P$ has no self-dependent cell. Define the ladder strip of $P$ to be the empty set;

2. Otherwise, denote $C_{0}$ the even self-dependent cell enclosed by $P$, which is labeled with the maximal integer. Define the ladder strip of $P$ to be the maximal sequence of cells $C_{0}, C_{1}, C_{2}, \ldots$, where $C_{2 i+1}$ is the adjacent cell to the East of $C_{2 i}$ and $C_{2 i+2}$ is the adjacent cell to the North-East of $C_{2 i+1}$ for each $i$.

Fig. 2 illustrates the $x$-labeling of a ternary path, whose ladder strip consists of the gray cells $C_{0}, C_{1}, C_{2}, C_{3}, C_{4}$ with labels $6,7,8,9,10$.

Suppose the ternary path $P$ has $k$ even self-dependent cells. We give the strip decomposition of $P$ recursively by the following steps:

1. If $k=0$, then the strip decomposition of $P$ is the empty set;

2. Otherwise, decompose $P$ into $P_{1} L_{1}$, where $L_{1}$ is the ladder strip of $P$ and $P_{1}$ is the ternary path obtained from $P$ by deleting $L_{1}$. We can associate $L_{1}$ with a sequence of simple transpositions, say $\sigma_{1}=s_{i} s_{i+1} \cdots s_{j}$, where $\{i, i+1, \ldots, j\}$ is the set of labels in $L_{1}$. Define $h_{1}:=i$, and $t_{1}:=j$; 
3. Repeat the above procedures for the ternary path $P_{1}$, we will get $\sigma_{2}$. Furthermore, we can find $\sigma_{3}, \ldots, \sigma_{k}$ by applying this step recursively. Then a set of parameters $\left\{\left(h_{i}, t_{i}\right) \mid 1 \leq i \leq k\right\}$ is obtained;

4. The strip decomposition of $P$ is $\sigma=\sigma_{1} \sigma_{2} \cdots \sigma_{k}$.

See Fig. 2 for an example, the ternary path

$$
P=E E E E E N E E E N E E N E N E N N
$$

can be decomposed into $P_{1} L_{1}$, where $P_{1}=$ EEEEENENEENEENEENN and $L_{1}$ is the ladder strip of $P$ with labels $6,7,8,9,10$. Thus $\sigma_{1}=s_{6} s_{7} s_{8} s_{9} s_{10}$. Moreover, the strip decomposition of $P$ is

$$
\sigma=\sigma_{1} \sigma_{2} \sigma_{3}=\left(s_{6} s_{7} s_{8} s_{9} s_{10}\right)\left(s_{4}\right)\left(s_{2} s_{3} s_{4} s_{5} s_{6} s_{7} s_{8} s_{9} s_{10} s_{11}\right) .
$$

Let $\Lambda=(1122 \cdots n n) \sigma$. Now we are led to the following results.

Lemma 4.1. $\Lambda$ is a matching, and $\sigma$ is the canonical reduced decomposition of $\Lambda$.

Proof. It suffices to show that $\sigma$ satisfies the conditions (4.1) and (4.2). The condition (4.2) for $\sigma$ is straightforward. To certify the condition (4.1) for $\sigma$, we first prove $h_{1}>h_{2}$.

Recall that $h_{2}$ is the label of an even self-dependent cell, denoted by $C$, enclosed by $P_{1}$. Obviously, $C$ is an even cell enclosed by $P$. We claim that $C$ is also self-dependent in $P$ : Otherwise, the adjacent cell, say $\widehat{C}$, to the South-West of $C$ is enclosed by $P$ but not by $P_{1}$. It implies that $\widehat{C}$ belongs to the ladder strip $L_{1}$ of $P$. Notice that $\widehat{C}$ is an odd cell. By the construction of $L_{1}, \widehat{C}$ is followed by the even cell $C$ in $L_{1}$. This contradicts that $C$ is enclosed by $P_{1}$.

By the above claim, $h_{2}$ is the label of an even self-dependent cell enclosed by $P$. Since $h_{1}$ is the maximal label of the even self-dependent cells in $P$, one sees that $h_{1} \geq h_{2}$. Observing that all the even self-dependent cells enclosed by $P$ have distinct labels, we deduce $h_{1}>h_{2}$. Recursively, the condition (4.1) is true for $\sigma$.

Lemma 4.2. $\Lambda$ is a 12312-avoiding matching.

Proof. By Lemma 4.1, it remains to show that $\sigma$ satisfies the condition (4.3).

Let $L_{i}$ and $L_{j}$ denote two ladder strips derived by the strip decomposition of $P$. In addition, the associated sequences of simple transpositions are $\sigma_{i}=s_{h_{i}} s_{h_{i}+1} \cdots s_{t_{i}}$ and $\sigma_{j}=s_{h_{j}} s_{h_{j}+1} \cdots s_{t_{j}}$ respectively. Assume that $i<j$. We have the following cases.

If $t_{j} \leq h_{i}-2$, the condition (4.3) follows immediately.

Otherwise, $t_{j} \geq h_{i}-1$. According to (4.1) and (4.2), we obtain $t_{j} \geq h_{i}-1>h_{j}$. By the construction of $L_{j}$, there is a cell, say $D$, in $L_{j}$ labeled with $h_{i}-1$. It follows that $D$ is an odd cell. Note that each cell enclosed by $P$ and touching the line $y=x / 2$ is even. Hence, $D$ is not a cell touching $y=x / 2$. This implies that the adjacent cell, say $\widehat{D}$, to the West of $D$ is enclosed by $P$. Moreover, $\widehat{D}$ is a cell in $L_{j}$. 
Let $B_{0}, B_{1}, B_{2}, \ldots$ and $\ldots, \widehat{D}, D, \ldots$ be the sequences of cells in $L_{i}$ and $L_{j}$, respectively. Since the cell $B_{0}$ is self-dependent and labeled with $h_{i}$, we derive that $B_{0}$ is in a column adjacent to $D$ and in a row not higher than $D$. See Figure 3 for the relative positions of cells in $L_{i}$ and $L_{j}$. Clearly, for each cell $B_{k}$ in $L_{i}$, there is a cell in $L_{j}$, denoted by $A_{k}$, which is in the same column as $B_{k}$. By the labeling rules, $A_{k}$ and $B_{k}$ have the same label. Therefore, the labels $t_{i}$ and $t_{j}$ of the ending cells in $L_{i}$ and $L_{j}$ must satisfy $t_{j} \geq t_{i}$. This completes the proof.

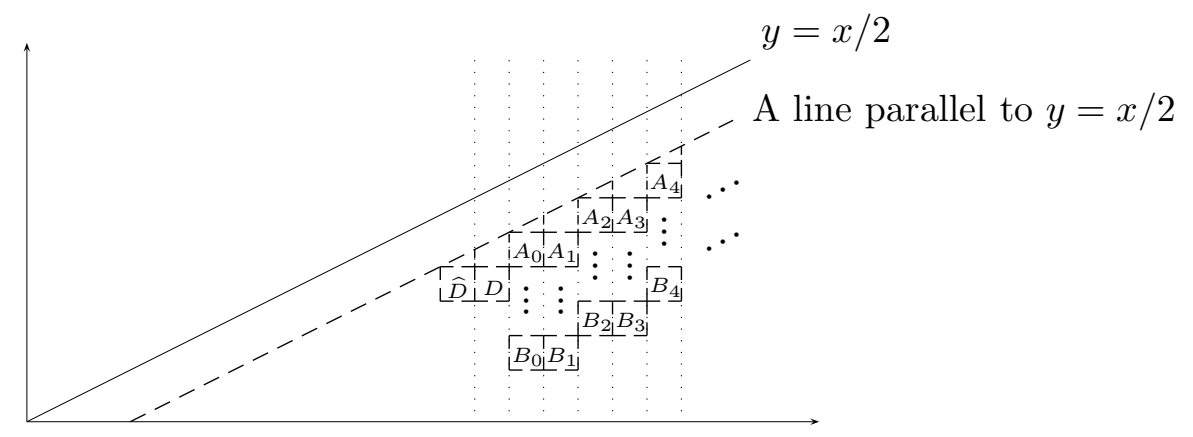

Figure 3: The relative positions of cells in $L_{i}$ and $L_{j}$.

Conversely, for a set of parameters $\left\{\left(h_{i}, t_{i}\right) \mid 1 \leq i \leq k\right\}$ satisfying the conditions (4.1)(4.3), one sees that the procedures are reversible to construct a ternary path. Therefore, we conclude with the following theorem.

Theorem 4.3. There is a bijection between the set of ternary paths of length $3 n$ and $\mathcal{M}_{n}(12312)$.

By the strip decomposition and Corollary 2.6, we easily derive the following result.

Corollary 4.4. For a ternary path $P$, the number of unit cells enclosed by $P$ equals the number of inversions in the corresponding matching.

\section{Acknowledgments.}

We would like to thank the referees for helpful suggestions to improve the presentation. This work was supported by the National Natural Science Foundation of China (Projects 11026050 and 11001073), the Natural Science Foundation of Hebei Province and Hebei University of Science and Technology (Project QD200956).

\section{References}

[1] J. Bandlow, E.S. Egge and K. Killpatrick, A weight-preserving bijection between Schröder paths and Schröder permutations, Ann. Comb. 6 (2002) 235-248.

[2] J. Bandlow and K. Killpatrick, An area-to-inv bijection between Dyck paths and 312-avoiding permutations, Electron. J. Combin. 8 (2001) \#R40. 
[3] S.C. Billey, W. Jockusch and R.P. Stanley, Some combinatorial properties of Schubert polynomials, J. Algebraic Combin. 2 (1993) 345-374.

[4] W.Y.C. Chen, Y.P. Deng and L.L.M. Yang, Motzkin paths and reduced decompositions for permutations with forbidden patterns, Electron. J. Combin. 9 (2003) \#R15.

[5] W.Y.C. Chen, T. Mansour and S.H.F. Yan, Matchings avoiding partial patterns, Electron. J. Combin. 13 (2006) \#R112.

[6] A. de Médicis and X.G. Viennot, Moments des q-polynômes de Laguerre et la bijection de Foata-Zeilberger, Adv. in Appl. Math. 15 (1994) 262-304.

[7] M. de Sainte-Catherine, Couplages et Pfaffiens en combinatoire, physique et informatique, Ph.D. Thesis, University of Bordeaux I, 1983.

[8] E.S. Egge and T. Mansour, Permutations which avoid 1243 and 2143, continued fractions, and Chebyshev polynomials, Electron. J. Combin. 9 (2003) \#R7.

[9] I.M. Gessel and X.G. Viennot, Determinants, paths and plane partitions, preprint, 1989.

[10] D. Gouyou-Beauchamps, Chemins sous-diagonaux et tableaux de Young, Combinatoire Enumerative (Montreal 1985), Lecture Notes in Math. 1234 (1986) 112-125.

[11] D. Gouyou-Beauchamps, Standard Young tableaux of height 4 and 5, European J. Combin. 10 (1989) 69-82.

[12] V. Jelínek, N.Y. Li, T. Mansour and S.H.F. Yan, Matchings avoiding partial patterns and lattice paths, Electron. J. Combin. 13 (2006) \#R89.

[13] M. Klazar, On $a b a b$-free and abba-free set partitions, European J. Combin. 17 (1996) $53-68$.

[14] M. Klazar, Bell numbers, their relatives, and algebraic differential equations, J. Combin. Theory Ser. A 102 (2003) 63-87.

[15] M. Klazar, Non-P-recursiveness of number of matchings or linear chord diagrams with many crossings, Adv. in Appl. Math. 30 (2003) 126-136.

[16] T. Mansour, E.Y.P. Deng and R.R.X. Du, Dyck paths and restricted permutations, Discrete Appl. Math. 154 (2006) 1593-1605.

[17] R. Simion and F.W. Schmidt, Restricted permutations, European J. Combin. 6 (1985) 383-406.

[18] N.J.A. Sloane, The On-Line Encyclopedia of Integer Sequences, http://www.research.att.com/ njas/sequences/.

[19] R.P. Stanley, On the number of reduced decompositions of elements of Coxeter groups, European J. Combin. 5 (1984) 359-372.

[20] P.R. Stein, On a class of linked diagrams, I. Enumeration, J. Combin. Theory Ser. A 24 (1978) 357-366.

[21] J. Touchard, Sur un problème de configurations et sur les fractions continues, Canad. J. Math. 4 (1952) 2-25. 